\title{
FALSE DIAGNOSIS IS A THREAT - A CASE STUDY
}

\author{
JAYASWAL RP* \\ Department of Medical Laboratory Technology, Amity Medical School, Amity University, Haryana 122 - 413, India. \\ Email: rpjayaswal07@gmail.com
}

Received: 12 June 2018, Revised and Accepted: 03 October 2018

\section{ABSTRACT}

Objective: To explore the possibility of false diagnosis \& threat to health.

Methods: In the present study, a case history of 23-year-old lactating woman was taken. Seventy-two test parameters were examined at SRL Lab Gurugram and Thyrocare Mumbai on alternative days from different location under variable climatic and family environment.

Results: The tests report of SRL lab was abnormal in range in compare to the reports of Thyrocare Lab.

Conclusion: The present study is expected to attract the attention of people who may be in the position of acquiring health services. In such approach, incidence of false-positive laboratory reports either due to technical errors or patient own conditions is thought to misguide a clinician who may prescribe wrong medicines to the patient. This type of mistake may invite unwanted and life-threatening threats to the patients' body if inappropriate or wrong drugs are given to the patient. Through this study, it is now suggested to be cautious and should verify before taking any drugs suggested by clinicians. Once a diagnosis is confirmed and considered repeatedly, the development of adverse health effects due to unwanted drugs can be minimized.

Keywords: Diagnosis, Treatment, Drugs, Laboratory reports.

(C) 2019 The Authors. Published by Innovare Academic Sciences Pvt Ltd. This is an open access article under the CC BY license (http://creativecommons. org/licenses/by/4. 0/) DOI: http://dx.doi.org/10.22159/ajpcr.2019.v12i2.27886

\section{INTRODUCTION}

Habitually, physician prescribes medicine based on the laboratory test report and in correlation with clinical symptoms and history. Sometimes, both clinical symptoms and laboratory findings may become wrong under stress and depression state, especially during and after pregnancy. Hereunder, a case is explained to aware each of us that false diagnosis may become a threat to the patient. In a case report, it was stated that, even though the technique is reliable and accurate, it may produce false-positive diagnosis under some circumstances [1].

\section{Patient history}

A 23-year-old lactating woman had symptoms of thyroid, anemia, weakness, and pain in bone and muscles. Her blood pressure was lower and pulse rate was higher. Her baby boy was 10 months old and normal in health. She was also having mild post-delivery depression.

\section{METHODS}

\section{Patient consent}

The patient consent was taken in written as well as in verbal communication to use her laboratory report in the present study.

\section{Specimen}

Blood and urine were taken.

\section{Sample collection}

Her first blood and urine samples were collected at Amity University, Gurgaon, and examined at SRL Laboratory, Gurugram. After the blood test, she went to her own home town due to the summer vacation and given blood samples to nearby diagnostic center after 1 week of her blood test to verify her earlier diagnosis. Her second blood sample was collected at Rupnarayanpur, West Bengal, and sent to Thyrocare, Mumbai, for examination. Both the samples were collected in fasting condition.

\section{Test parameters and methods (Table 1)}

Seventy-two test parameters were taken for laboratory investigation to assess her general health, thyroid, diabetes, bone, lipid, kidney, liver, fatigue, and muscles related problems. The following methods were used to perform the specific tests:

a. Complete blood count: Coulter impedance principle, photometric measurement, calculated parameter, and derived parameter

b. Differential count: VCS Technology and ethylenediaminetetraacetic acid smear

c. Erythrocyte sedimentation rate: Automated (photometrical capillary stopped-flow kinetic analysis)

d. Peripheral smear examination: Microscopic examination

e. Urine analysis: Dip stick/microscopy/reflectance spectrophotometry

f. Serum iron, total iron binding capacity, glucose fasting, glycosylated hemoglobin, mean plasma glucose, bilirubin total/direct, total protein, albumin, globulin, albumin: globulin ratio, aspartate aminotransferase, alanine aminotransferase, alkaline phosphatase, gamma-glutamyl transferase, lactate dehydrogenase, calcium, cholesterol (CHOL), triglycerides, high-density lipoprotein (HDL) CHOL, direct low-density lipoprotein (LDL) CHOL, non-HDL CHOL, CHOL: HDL ratio and LDL: HDL ratio, very LDL, blood urea nitrogen (BUN), creatinine, BUN: Creatinine ratio, uric acid, magnesium, electrolytes (NA/K/CL): Spectrophotometry (automated)

g. Thyroid panel, 25-Hydroxyvitamin D and Vitamin B12: Chemiluminescence.

\section{RESULTS}

After receiving test report from SRL Diagnostic laboratory, it was observed that most of the test values were in abnormal range (Table 1). She did not consulted any clinicians after her first blood checkup, and hence, she did not take any medicines also.

In her second blood reports, she noticed miraculous findings. All the test values which were abnormal in her previous report become normal. 
Table 1: First laboratory investigation report interpretation

\begin{tabular}{ll}
\hline Test & Value \\
\hline RBC count & Elevated \\
Red cell distribution & \\
width & \\
Mean platelet volumes & \\
ESR & \\
Mean plasma glucose & \\
Alkaline phosphatase & \\
Lactate dehydrogenase & \\
TSH third generation & \\
MCV & Lowered \\
MCH & \\
MCHC & \\
25-Hydroxyvitamin D & \\
CHOL/HDL ratio & \\
Total protein & Near upper reference range \\
Sodium & \\
Potassium & \\
Chloride & \\
Calcium & \\
Creatinine & \\
Peripheral blood smear & Predominantly microcytic \\
& \\
HbA1C & anisocytosis and mild erythrocytosis \\
\hline
\end{tabular}

TSH: Thyroid-stimulating hormone, HbA1C: Glycosylated hemoglobin, RBC: Red blood cell, CHOL: Cholesterol, HDL: High-density lipoprotein, ESR: Erythrocyte sedimentation rate, MCV: Mean corpuscular volume, MCH: Mean corpuscular hemoglobin, MCHC: Mean corpuscular hemoglobin concentration

\section{Observation}

SRL diagnostics laboratory is one of the reputed laboratories in India. Their test reports are assumed reliable and trusted by millions. From the patient's report, it was found that many test parameter results were abnormal. Based on the diagnosis, it is certain that clinicians can prescribe medications to normalize thyroid, liver, and many other indicated diagnoses. Instead in her second report, there was no any suggested diagnosis of being sick. Similarly, thyrocare is also a one of the India's leading diagnostic laboratories that have excellent quality control and management system. Their test reports are also trusted by millions. Both of these laboratories use recent technology during the procedure of diagnosis.

Now, it was time for big debate that how the report becomes normal without taking medicine.

\section{DISCUSSION}

In the earlier report, her sample was collected when she was in Gurugram at extreme temperate climate around $44-47^{\circ} \mathrm{C}$. During the night, she fed her baby repeatedly and she did not drink water till the morning. She was having depression, incomplete sleep, and stress due to her baby. She was not able to take meal rich in nutrients including milk, curd, and other supplements. All these factors made her extremely dehydrated, weak, and stressful. Hence, most of her test results were abnormal $[2,3]$.

Concerning her second report, her blood sample was collected after 1 week at Rupnarayanpur, West Bengal, India, where the climate zone was humid with temperature $33-35^{\circ} \mathrm{C}$. Although she fed her baby in similar way, she had full comfort life and good care for herself and baby too by family members. No dehydration and depression were noticed, and she was happy and having normal sleep too.
She was taking normal diet containing milk and curd which might have brought homeostasis due to which she becomes normal $[4,5]$.

Referring to the first report, if treatment would have been initiated, she further might have developed side effects and complications due to therapeutic drugs.

It has been documented that disturbed sleep patterns and significant fatigue during breastfeedings cause disbalance in blood profile [6,7]. Furthermore, lipid profile may increase if the sample is collected in dehydrated condition [8].

In another studies, the fatigue during breastfeeding also has been documented to activate immune response [9].

\section{CONCLUSION}

From the above studies, it may be noticed that dehydration, depression, and incomplete rest or sleep are the major factors for variations in her blood reports.

Hence, it is suggested that, before taking any medicine, each of us has to ensure that there are no dehydration, depression, and lack of rest. These are interfering factors which leads to misdiagnosis and mistreatment and may invite life-threatening conditions due to the intake of the prescribed drugs.

To prevent this mishappening, it is suggested to know the root cause of abnormal report. It is suggested to make a change in the lifestyle and/ or location of stay; to get away from current busy schedules and seek full support and care of family members. The approach of rehabilitation efforts are expected to produce effective changes in the personal health. Instead of those practice, if anyone starts to take medicines as a stimulant or inhibitors, there can be the possibility of adverse health effects. Slowly, the action of drugs can further invite life-threatening conditions through organ damage/failure.

\section{AUTHORS' CONTRIBUTIONS}

As a sole author, I had participated sufficiently in the work to take public responsibility for the content, including participation in the concept, design, analysis, writing, or revision of the manuscript.

\section{CONFLICTS OF INTEREST}

The author declares no conflicts of interest.

\section{REFERENCES}

1. Vachiéry JL, Brimioulle S, Crasset V, Naeije R. False-positive diagnosis of pulmonary hypertension by doppler echocardiography. Eur Respir J 1998; $12: 1476-8$.

2. Alomar M. Factors affecting postpartum depression among women of the UAE and Oman. Int J Pharm Pharm Sci 2015;7:231-3.

3. Rabbani D. Depression in pregnancy - Consequences and treatment modalities. Int J Pharm Pharm Sci 2016;8:24-7.

4. Jayaswal RP, Prabhakar PK. Probiotics- A new diabetes management tool. Int J Green Pharm 2017;11:S395.

5. Jayaswal RP, Vijayasimha M, Prabhakar PK. Gut microbiota and diabetes mellitus - An interlinkage. Asian J Pharm Clin Res 2018;11:13-6.

6. Gay CL, Lee KA, Lee SY. Sleep patterns and fatigue in new mothers and fathers. Biol Res Nurs 2004;5:311-8.

7. Wambach KA. Maternal fatigue in breastfeeding primiparae during the first nine weeks postpartum. J Hum Lact 1998;14:219-29.

8. Campbell NR, Wickert W, Magner P, Shumak SL. Dehydration during fasting increases serum lipids and lipoproteins. Clin Invest Med 1994;17:570-6.

9. Almas S, Vance J, Baker T, Hale T. Management of multiple sclerosis in the breastfeeding mother. Mult Scler Int 2016;2016:6527458. 